\title{
Treatment and Management of Depression Symptoms in Pregnant Veterans: Varying Experiences of Mental Health Care in the Prenatal Period
}

\author{
Aimee R. Kroll-Desrosiers ${ }^{1,6}$ (D) Sybil L. Crawford ${ }^{2}$ - Tiffany A. Moore Simas ${ }^{1,3,4,5}$ • \\ Melissa A. Clark ${ }^{1} \cdot$ Kristin M. Mattocks ${ }^{1,6}$
}

Published online: 01 February 2020

(C) This is a U.S. government work and not under copyright protection in the U.S.; foreign copyright protection may apply 2020

\begin{abstract}
Depression screening is recommended for all pregnant veterans; however, little is known on how often symptomatic women receive care, how depression treatment presents in practice, and whether women veterans are utilizing treatment during the appreciable perinatal period. Our sample included 142 pregnant veterans from 15 Veterans Health Administration (VA) medical facilities with Edinburgh Postnatal Depression Scale (EPDS) scores $\geq 10$. Sociodemographic characteristics, military service, health utilization, and pregnancy related factors were collected as part of a telephone survey. A majority of our sample (70\%) had 1 or more mental health visits or antidepressant prescriptions during pregnancy. Women with a history of depression had more mental health visits and a higher percentage of antidepressant use before and during pregnancy than women without a history of depression. Pregnant women veterans without a history of depression may be less likely to receive care for depression during pregnancy. However, the majority of our veterans showing depression symptoms prenatally had at least one mental health visit or an antidepressant medication fill during their pregnancy window, suggesting that mental health care is readily available for women veterans.
\end{abstract}

Keywords Veterans $\cdot$ Pregnancy $\cdot$ Mental health $\cdot$ Depression

Aimee R. Kroll-Desrosiers

aimee.kroll-desrosiers@va.gov

1 Department of Population and Quantitative Health Sciences, University of Massachusetts Medical School, 368 Plantation Street, Worcester, MA 01605, USA

2 Graduate School of Nursing, University of Massachusetts Medical School, Worcester, MA, USA

3 Department of Obstetrics \& Gynecology, University of Massachusetts Medical School/UMass Memorial Health Care, Worcester, MA, USA

4 Department of Pediatrics, University of Massachusetts Medical School, Worcester, MA, USA

5 Department of Psychiatry, University of Massachusetts Medical School, Worcester, MA, USA

6 Research and Development, VA Central Western Massachusetts, Leeds, MA, USA 


\section{Background}

Depression is common during the prenatal period among women veterans [1]. Women veterans are more likely to experience combat-related trauma and post-deployment stressors, and in general have higher rates of depression, compared to the general population [2]. A recent prospective study identified depression symptoms in nearly $30 \%$ of pregnant veterans [3]. Despite high rates of depression, there is little understanding of how pregnant veterans manage their mental healthcare during pregnancy, especially important because the perinatal time marks a distinct period when women encounter their providers on a regular basis, offering unique opportunities for screening and treatment for depression [4].

Because women remain a numerical minority in the Veterans Health Administration (VA), VA medical facilities do not provide onsite maternity care. Rather, maternity care for veterans is provided by non-VA obstetricians and reimbursed by the VA. The VA recommends depression screening for all pregnant veterans [5], however, there is a paucity of research regarding the frequency or quality of depression treatment during pregnancy. Previous studies suggest that while receiving prenatal care from non-VA providers, a substantial proportion of veterans concurrently receive mental health care within the VA $[1,6]$. Coordination of care between non-VA and VA providers is an important aspect of maternity care for veterans. VA guidelines state that information sharing, "particularly in the area of medication management and monitoring for medications that have teratogenic effects," is "critical to patient safety" [5]. Coordination of medication risk discussions between the patient, her mental health provider, and her obstetrician has previously been shown to contribute to greater depression treatment during the perinatal period $[7,10,20]$.

Psychotherapy has been shown to be an effective treatment strategy for pregnant women with depression [8,9]. However, therapy brings its own challenges, among them patient barriers such as cost, travel difficulties, and time constraints [9], as well as systematic barriers such as lack of provider training, limited access to providers, and absence of standardized screening and referral processes [10].

To date, few studies have examined pregnant veterans' use of antidepressants. The prevalence rate of antidepressant use during pregnancy has been estimated to be near 5\% in the general population $[11,12]$, with some point estimates placing antidepressant use at any time during pregnancy close to $13 \%$ [13]. Little is known about the rates of antidepressant use among pregnant veterans; however, a previous study reported nearly $90 \%$ of the sample of female veterans of childbearing age had used any prescription medication within the past year [14]. Antidepressant use during pregnancy is not without risk, with associations between antidepressant use and miscarriage, low birthweight, preterm birth, and the risk for complications in newborns such as irritability, tachypnea, hypoglycemia, and temperature instability in the days following birth $[9,15]$. However, the risks of untreated maternal depression include behavior problems and cognitive delays in infants, comprised care giving practices, reduced rates of breastfeeding, maternal sleep problems, and greater suicidal ideation compared to women without depressive symptoms [16, 17]. Both the VA and the American College of Obstetricians and Gynecologists (ACOG) recommend risk/benefit discussions between a patient and her provider regarding continuing or starting a functional antidepressant medication during pregnancy $[15,18,19]$; however, rates as low as $24 \%$ have been reported for discussions between reproductive age female veterans and their providers about medication risks [14]. These challenges of available treatment options and the overall low adherence rate to treatment among depressed pregnant and postpartum women [20] have yet to be studied among veterans. 
Using a mixed-methods approach, our objective was to evaluate the rate of mental health care treatment utilization during pregnancy and to understand the experience and management of depression in a sample of pregnant veterans. We identify differences in treatment utilization during pregnancy among women who report past diagnoses of depression compared to those with no past diagnosis. Additionally, we explore the decisions women make regarding antidepressant medication use during pregnancy and the extent to which pregnant veterans discuss medication risk counseling with their VA and/or obstetrical providers.

\section{Methods}

\section{Study Design, Data Source, and Study Sample}

Participants were identified at 15 VA sites across the US as part of the Center for Maternal and Infant Outcomes Research in Translation (COMFORT) study [3, 21]. COMFORT was approved by the Veterans Administration Central Institutional Review Board (CIRB) and was given a waiver of consent by the $\mathrm{CIRB}$ so that signed consent forms were not required from participants; however, verbal informed consent was obtained from all individual participants included in the study. Pregnant veterans were identified by the veteran's VA provider. To utilize maternity care benefits, women veterans must have a visit with their VA provider to confirm their pregnancies prior to obtaining maternity care from an accredited prenatal provider [5]. All veterans confirmed to be pregnant were mailed recruitment packets that invited the veteran to call a toll-free number to receive further information. Additionally, research team members followed up the mailings with telephone calls to potential participants. Two telephone surveys were scheduled with pregnant veterans who agreed to participate; the first during the second or third trimester (around 20-24 weeks of gestation) and a second within three months of delivery.

Participants were reimbursed \$25 for each survey. Enrollment rates were roughly 40\%; of the 1410 eligible women who were sent invitation letters, 562 had agreed to participate at the time of this analysis. Quantitative study data were collected and managed using REDCap (Research Electronic Data Capture), a secure, web-based application designed to support data capture for research studies [22]. Additionally, electronic health record (EHR) data on date of birth, service-connected disability status, past medical diagnoses, and healthcare utilization during pregnancy were obtained on participants from VA Corporate Data Warehouse (CDW) administrative data. The CDW is a repository of data updated nightly from the VA electronic medical records system for operations and research use [23].

Our sample included 142 participants enrolled within the first two years of the study (January 28, 2016 - February 6, 2018) and scoring $\geq 10$ on a prenatal Edinburgh Postnatal Depression Scale (EPDS); we were able to match 135 (95\%) participants to their EHR within the VA CDW. Of those missing CDW data, the majority $(n=5,71 \%)$ did not provide their social security number or only provided the last 4 digits, preventing identification in the CDW data. The remaining participants $(n=2)$ could not be matched with a social security number in the CDW.

\section{Definition of Quantitative Outcomes}

Healthcare utilization was examined through mental health visits and antidepressant use, both as recorded in VA EHR data and self-reported in the prenatal COMFORT survey. We 
examined healthcare utilization in two time-periods: pre-pregnancy and pregnancy. We defined pregnancy as 280 days prior to the newborn date of birth (available for $59 \%$ of our sample) or estimated delivery date (for the remaining $41 \%$ of our sample), as done in previous analyses $[24,25]$. Pre-pregnancy was considered prior to the start of the pregnancy time period, defined as 280 to 560 days prior to delivery or estimated due date (newborn date of birth-280 days)280 days) (Fig. 1). This gave us comparable time periods to examine healthcare utilization in our sample prior to and during pregnancy.

To identify mental health care visits, we utilized VA mental health stop codes, indicators in the EHR that flag the clinical group responsible for an outpatient encounter (Online Resource A) [26]. We also obtained information on receipt of an antidepressant medication, where a 30day fill prescribed at any point during our pre-pregnancy or pregnancy windows was considered receipt of a prescription antidepressant. We opted to include only antidepressant prescriptions because we specifically asked about antidepressant use in our survey and used the EPDS to determine depression symptomology. Our definition of antidepressants included selective serotonin reuptake inhibitors (SSRI), serotonin-norepinephrine reuptake inhibitors (SNRI), tricyclic antidepressants (TCA), monoamine oxidase inhibitors (MAOI), noradrenergic and specific serotonergic antidepressants (NASSA), norepinephrine-dopamine reuptake inhibitors (NDRI), bupropion, selective serotonin reuptake enhancers (SSRE), tianeptine, norepinephrine-dopamine disinhibitors (NDDI), and agomelatine (Online Resource B). We created an additional variable, "Any VA Mental Health Care during Pregnancy" defined as one or more mental health visits during pregnancy or any receipt of an antidepressant prescription.

Additionally, we examined self-reported mental health care utilization using responses from the prenatal survey. Participants were asked about antidepressant use with the following questions: "Before this recent pregnancy, had you ever been prescribed anti-depressants?" immediately followed by "Currently, are you taking any medications for mental health conditions?". To identify visits with a mental health provider, participants were asked: "At

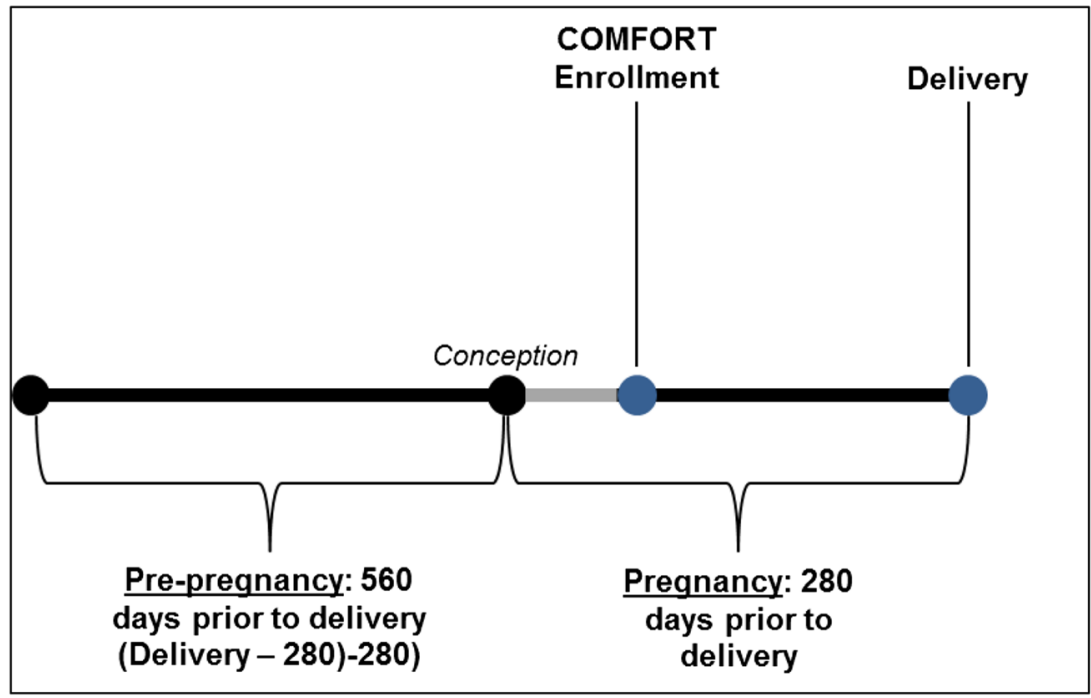

Fig. 1 Time Periods of Mental Health Care/Antidepressant Prescription Identification from VA Electronic Health Records 
the time you found out you were pregnant were you seeing a mental health provider?" and "During this pregnancy will you continue to see a mental health provider?"

\section{Demographic, Military, Health-Related, and Pregnancy Factors}

We selected potential correlates of depression care utilization during pregnancy based on previous literature, including history of depression [27], as well as data available from the COMFORT telephone survey. The telephone survey collected information including sociodemographics, military service, health status, healthcare utilization, social support, and pregnancy related factors. Participants were asked if they had ever been diagnosed with depression. A 10-item Edinburgh Postnatal Depression Scale (EPDS) was also administered [28], with a cutoff of 10 used to define present depression symptoms [29] and a cutoff of 13 used to suggest probable major depression [30]. Multiple times during the interview, participants were reminded that they could refuse further participation in the study or refuse to answer specific questions. Participants with affirmative responses to the self-harm question of the EPDS were directed to further clinical follow-up.

Data used in this analysis included demographics (race/ethnicity, marital status, and employment status), military characteristics (deployment history and history of military sexual trauma (MST), adapted from the VA's universal MST screening questions and described in previous literature [31]), as well as self-reported past mental health diagnoses from the COMFORT survey. From the EHR, past depression diagnoses were identified as ever being present by the following ICD-9 and ICD-10 diagnosis codes: ICD-9: 293.83, 296.2, 296.3, 296.90, 298.0, 300.4, 309.0, 309.1, 311; ICD-10: F32, F33, F34, F39. To examine the reliability and validity of self-reported depression diagnoses in this sample of veterans, we performed a reliability analysis using Cronbach's $\alpha$ and calculated sensitivity, specificity, and positive predictive values to compare to EHR variables for the same diagnoses. We found sensitivity, specificity, and positive predictive values rates of $0.90,0.42$, and 0.77 , respectively, with most discordant cases for women self-reporting the condition when it was absent in the EHR (data not shown). Age, OEF/OIF status, and service-connected status were also obtained from EHR data. OEF/OIF status included any veteran who served in any of those specific military tours; service-connected disability status was determined by the Veteran Benefits Administration and measured as a percentage from $0 \%$ to $100 \%$, where $0 \%$ is the lowest level of disability and is different from not having a disability [32]; here dichotomized as presence of service-connected disability percentage of yes $(>0 \%)$ or no as in previous analyses [24].

\section{Qualitative Methods}

Prior to the start of recruitment, we developed a semi-structured interview guide with questions related to the experiences of veterans regarding depression management and other mental health care during pregnancy (Online Resource C). All enrolled veterans participated in the semi-structured interview. We selected a purposive sample of 42 interviews from participants with a prenatal EPDS $\geq 10$ in which medication management or mental health care were discussed specifically; these interviews were selected from all interviews with pregnant veterans showing depressive symptoms.

The open-ended responses of the interview were digitally recorded to capture verbatim content and were professionally transcribed for this analysis. Interview notes and recordings were linked by an unidentifiable participant code. 


\section{Analysis}

\section{Quantitative Analysis}

Descriptive statistics, including means, standard deviations, and ranges for continuous variables, and frequencies for categorical variables, were calculated and presented.

To examine correlates of any VA mental health care during pregnancy, we conducted bivariate analyses of variables of interest by our derived mental health care variable of one or more mental health visits during pregnancy or any receipt of an antidepressant prescription. Chi-squared tests and relative risks with $95 \%$ confidence intervals were calculated.

As we additionally sought to learn more about how women veterans treated and managed their depression symptoms during pregnancy, we then compared demographic, military, and mental health characteristics by women who reported having been diagnosed with depression prior to pregnancy versus those who reported no past diagnosis. We used chi-squared tests to examine differences in categorical factors and Student's t test to examine mean differences in continuous factors.

We also ran sensitivity analyses to test the reliability of our 280-day pregnancy window. We calculated estimated gestational age at delivery in our sample, using the difference between the date of the prenatal COMFORT interview and self-reported gestational age at that interview to set a "time zero". We then calculated the difference between "time zero" and delivery date or estimated due date. Our average estimated gestational age at delivery was 275.25 days ( \pm standard deviation of 10.44) with a median and mode of 280 days. Our shortest estimated gestational period was 203 days; our longest estimated gestational period was 296 days. Given our minimum and maximum estimated gestational window of 203 days and 296 days, we reran our calculations of mental health visits and antidepressant prescriptions for pre-pregnancy and pregnancy windows of 200 and 300 days, respectively (Appendices D \& E).

All quantitative analyses were conducted in SAS version 9.2 (SAS Institute, Inc., Cary, North Carolina).

\section{Qualitative Analysis}

We utilized a phenomenological approach to understand how pregnant veterans managed their depression symptoms and mental health care during pregnancy. Phenomenological approaches are used to understand situational experiences viewed through the personal perceptions of participants $[33,34]$, requiring the researchers to consider the analysis without preconceived ideas or beliefs about the experiences of the participants [35]. Guided by this approach, we conducted an exploratory, inductive analysis similar to methods from previous work [36]. This type of analysis is wellsuited for our semi-structured interview data focusing on pregnancy, where we interpreted participant responses through an iterative process [33, 34].

We used open coding, where one main coder (ARKD) read participant transcripts lineby-line, forming initial notes and definitions and then objectively organized personal narratives into similar experiences, grouping by phrase and keywords. An additional coder (KM) reviewed these initial codes for clarity and qualitative accuracy. Next, we interpreted and refined the experiences shared by pregnant veterans and worked to understand the connections between emergent themes and their descriptions of how these experiences are shaped. Findings were organized by clusters of superordinate and 
subthemes in a coherent order, retranslated into a narrative account, and considered in relation to quantitative results. Qualitative analysis was managed in ATLAS.ti version 8.2.30 (Scientific Software GmbH, Berlin, Germany).

\section{Results}

\section{Quantitative Results}

Women in our sample were on average 31.4 years of age $( \pm 4.5)$, married $(50.7 \%)$, white $(52.1 \%)$, and deployed in the past $(57.7 \%)$. Prenatal EPDS scores averaged $14.7( \pm 4.0)$ (Table 1). Overall, the number of mental health visits increased slightly from the prepregnancy to pregnancy time periods, from 5.3 visits in the 280 days prior to pregnancy, to 6.1 visits during the 280-day pregnancy window (Wilcoxon signed-rank test $p=0.18$ ). Antidepressant use decreased from $40.1 \%$ in pre-pregnancy to $37.0 \%$ of our sample having received a similar prescription during pregnancy (McNemar's test for paired samples $p=0.21$ ).

A majority of our sample $(69.7 \%)$ had at least 1 mental health visit or antidepressant prescription during pregnancy (Table 1). Past treatment for military sexual trauma (MST) $\left(\chi^{2}\right.$

Table 1 Participant Characteristics $(n=142)$

\section{COMFORT Survey Data}

Age at Prenatal Interview (Mean $\pm \mathrm{SD}$, Range)

Married (N, \%)

Race: White (N, \%)

Hispanic or Latino/Latina (N, \%)

Past Deployment (N, \%)

$\mathrm{OEF} / \mathrm{OIF}(\mathrm{N}, \%)$

Prenatal EPDS (Mean \pm SD, Range)

Symptoms of Probable Major Depression, EPDS $\geq 13$ (N, \%)

Weeks Pregnant at Prenatal Interview (Mean \pm SD, Range)

Care Utilization from VA CDW Electronic Health Record*

Mental Health visits, mean \pm SD (range)

Pre-pregnancy

Pregnancy

Any Mental Health visits, n (\%)

Pre-pregnancy

Pregnancy

Antidepressant Prescription, n (\%)

Pre-pregnancy

Pregnancy

Any VA Mental Health Care during Pregnancy (1+ mental health visit or antidepressant prescription in VA CDW EHR) $(\mathrm{N}, \%)$
Total

$31.4 \pm 4.5$

$(22.4-41.7)$

$72(50.7)$

$74(52.1)$

22 (15.5)

82 (57.7)

134 (94.4)

$14.7 \pm 4.0$

(10.0-26.0)

90 (63.4)

$23.0 \pm 5.3$

(4.4-39.1)

$5.3 \pm 9.1$

(0-45)

$6.1 \pm 9.4$

(0-54)

79 (55.6)

91 (64.1)

57 (40.1)

$50(35.2)$

99 (69.7)

CDW: Corporate Data Warehouse; EPDS: Edinburgh Postnatal Depression Scale; OEF/OIF: Operation Enduring Freedom/Operation Iraqi Freedom; VA: Veterans Affairs

Time periods calculated as Pre-pregnancy = (Newborn date of birth-280)-280; Pregnancy $=$ Newborn date of birth-280 $*_{n}=7$ participants were missing $C D W$ data 
$p=0.002)$ and drug abuse or alcoholism $\left(\chi^{2} p=0.004\right)$, self-reported diagnosis of depression $\left(\chi^{2} p=0.0002\right)$, PTSD $\left(\chi^{2} \mathrm{p}=0.002\right)$, mood disorder $\left(\chi^{2} p=0.009\right)$, and bipolar disorder (Fisher's Exact Test $p=0.02)$, past use of antidepressants $\left(\chi^{2} p<.0001\right)$, and established care with a VA mental health provider prior to pregnancy $\left(\chi^{2} \mathrm{p}<.0001\right)$ were significantly associated with at least 1 mental health visit or antidepressant prescription during pregnancy. Employment was associated with a decreased likelihood of a mental health visit or antidepressant prescription during pregnancy $\left(\chi^{2} p=0.03\right)$ (Table 2$)$.

A large proportion of our sample $(81.7 \%)$ reported a past diagnosis of depression. Those reporting a history of depression were statistically more likely to score a prenatal EPDS $\geq 13$, suggestive of major depressive symptoms $(68 \%$ vs. $42 \%, p=0.01)$. Women reporting no history of depression were more likely to be employed ( $54 \%$ vs. $32 \%, p=0.04$ ), less likely to report treatment for military sexual trauma ( $19 \%$ vs. $43 \%, p=0.02)$, and less likely to report past diagnoses of PTSD, and bipolar, mood, and anxiety disorders (all $p<0.05$ ) (Table 3 ).

Care utilization was higher in women with a self-reported history of depression, with a greater number of mental health visits before and during pregnancy and a higher percentage of antidepressant use during these same time periods, with $75.9 \%$ of women with a history of depression having some VA mental health care during pregnancy, compared to only $42.3 \%$ of women with no history of depression $(p<0.001)$ (Table 3; Figs. 2 and 3$)$. This corresponded to women with a self-reported history of depression being 1.8 times more likely to receive at least one VA mental health visit or antidepressant prescription during pregnancy than women without a history of depression (data not shown; relative risk $=1.8,95 \%$ confidence interval $=$ 1.1-2.8).

Our sensitivity results indicated that the proportion of our sample with CDW data $(n=135)$ who had mental health visits and/or antidepressant prescriptions varied depending on the pregnancy window we used (200-days, 280-days, or 300-days). The number of mental health visits increased as time windows were broadened. Receipt of an antidepressant prescription was present in $21.5 \%$ of women when examining a 200 -day pregnancy window; when that window was expanded to 280 -days and 300-days, $37 \%$ and $40 \%$ of women had received an antidepressant prescription, respectively. Women receiving at least one VA mental health visit

Table 2 Factors Significantly Associated with Any VA Mental Health Care during Pregnancy* $(n=135)$

\begin{tabular}{|c|c|c|c|c|}
\hline & $\mathrm{X}^{2}, p$ value & Relative Risk & $\begin{array}{l}\text { Lower } \\
95 \% \mathrm{CI}\end{array}$ & $\begin{array}{l}\text { Upper } \\
95 \% \mathrm{CI}\end{array}$ \\
\hline Employed vs. Unemployed/Homemaker/Student/Other & $4.99,0.03$ & 0.41 & 0.19 & 0.91 \\
\hline Treatment for Military Sexual Trauma (Yes vs. No) & $9.90,0.002$ & 4.34 & 1.66 & 11.35 \\
\hline Ever Treatment for Drug Abuse or Alcoholism (Yes vs. No) & $8.54,0.004$ & - & - & - \\
\hline Ever Depression Diagnosis (Yes vs. No) & $13.50,0.0002$ & 5.09 & 2.03 & 12.74 \\
\hline Ever PTSD Diagnosis (Yes vs. No) & $9.83,0.002$ & 3.45 & 1.56 & 7.62 \\
\hline Ever Mood Disorder Diagnosis (Yes vs. No) & $6.90,0.009$ & 4.78 & 1.36 & 16.81 \\
\hline Ever Bipolar Disorder Diagnosis (Yes vs. No) & $5.68,0.01^{* *}$ & - & - & - \\
\hline Ever Antidepressant Prescription Prior to Pregnancy (Yes vs. No) & $17.43,<.0001$ & 6.36 & 2.51 & 16.12 \\
\hline Plan to See VA MH Provider during Pregnancy (Yes vs. No) & $34.43,<.0001$ & 35.44 & 7.44 & 168.73 \\
\hline \multicolumn{5}{|c|}{$* 1+$ mental health visit or antidepressant prescription in VA CDW EHR } \\
\hline \multicolumn{5}{|l|}{ **Fisher's Exact Test for small cell sizes } \\
\hline \multicolumn{5}{|c|}{$\begin{array}{l}\text { ***No risk estimate computed for Ever Treatment for Drug Abuse or Alcoholism or Ever Bipolar Disorder } \\
\text { Diagnosis due to no self-report of either past condition in the "no VA care during pregnancy" group }\end{array}$} \\
\hline \multicolumn{5}{|c|}{$\begin{array}{l}C I=\text { confidence interval; } P T S D=\text { post-traumatic stress disorder; VA MH=Veterans Affairs Mental Health; } n=7 \\
\text { participants were missing } C D W \text { data }\end{array}$} \\
\hline
\end{tabular}


Table 3 Participant Characteristics by Self-Reported Past Depression Diagnosis $(n=142)$

\begin{tabular}{|c|c|c|c|}
\hline Characteristic & $\begin{array}{l}\text { No History } \\
\text { of Depression } \\
(N=26)\end{array}$ & $\begin{array}{l}\text { History } \\
\text { of Depression } \\
(N=116)\end{array}$ & $P$ value \\
\hline Probable Symptoms of MDD (Prenatal EPDS $\geq 13$ ) (N, \%) & $11(42.3)$ & $79(68.1)$ & 0.01 \\
\hline First Pregnancy $(\mathrm{N}, \%)$ & $10(38.5)$ & $32(27.6)$ & 0.27 \\
\hline \multicolumn{4}{|l|}{ Current Employment Status (N, \%) } \\
\hline Employed & $14(53.8)$ & $37(31.9)$ & 0.04 \\
\hline Unemployed/Homemaker/Student/Other & $12(46.2)$ & $79(68.1)$ & \\
\hline Service-Connected Status $(\mathrm{N}, \%)$ & $22(84.6)$ & $103(88.8)$ & $0.39 *$ \\
\hline Ever Counseling/Treatment for Military Sexual Trauma (N, \%) & $5(19.2)$ & $50(43.1)$ & 0.02 \\
\hline Ever received medical treatment for drug abuse or alcoholism (N, \%) & $1(3.8)$ & $20(17.2)$ & $0.12 *$ \\
\hline \multicolumn{4}{|l|}{ Self-Reported Mental Health Diagnoses, Ever Diagnosed (N, \%) } \\
\hline Bipolar Disorder & $0(0.0)$ & $16(13.8)$ & $0.04 *$ \\
\hline Other Mood Disorder & $0(0.0)$ & $35(30.2)$ & 0.001 \\
\hline Anxiety Disorder & $8(30.8)$ & $88(75.9)$ & $<.0001$ \\
\hline PTSD & $9(34.6)$ & $79(68.1)$ & 0.002 \\
\hline Schizophrenia & $0(0.0)$ & $1(0.9)$ & 0.99 \\
\hline $\mathrm{ADHD}$ or $\mathrm{ADD}$ & $0(0.0)$ & $13(11.2)$ & $0.13^{*}$ \\
\hline Eating Disorder & $0(0.0)$ & $10(8.6)$ & $0.21 *$ \\
\hline Self-Reported Ever Diagnosis of Any Mental Health Condition** (N, \%) & $14(53.8)$ & $116(100.0)$ & $<.0001 *$ \\
\hline $\begin{array}{l}\text { Self-Reported Ever Diagnosis of Any Mental Health Condition**, } \\
\text { excluding depression (N, \%) }\end{array}$ & $14(53.8)$ & $94(81.0)$ & 0.003 \\
\hline \multicolumn{4}{|l|}{ Care Utilization } \\
\hline Seeing MH provider when found out about pregnancy, self-reported (N, \%) & $7(26.9)$ & $62(53.4)$ & 0.03 \\
\hline Planned to see $\mathrm{MH}$ provider during pregnancy, self-reported $(\mathrm{N}, \%)$ & $7(26.9)$ & $68(58.6)$ & 0.36 \\
\hline Any VA Mental Health Visits Pre-pregnancy (N, \%) & $7(26.9)$ & $72(62.0)$ & 0.0006 \\
\hline Any VA Mental Health Visits during 280-Day Pregnancy Window (N, \%) & $11(42.3)$ & $80(69.0)$ & 0.006 \\
\hline $\begin{array}{l}\text { VA Mental Health Visits during 280-Day Pregnancy Window } \\
\text { (Mean } \pm \text { SD, Range) }\end{array}$ & $\begin{array}{l}2.7 \pm 4.7 \\
(0-18)\end{array}$ & $\begin{array}{l}6.9 \pm 10.1 \\
(0-54)\end{array}$ & $0.003 *$ \\
\hline Ever Prescribed Anti-Depressants, self-reported (N, \%) & $10(38.5)$ & $107(92.2)$ & $<.0001 *$ \\
\hline $\begin{array}{l}\text { Mental health medication use at the time of prenatal interview, self-reported } \\
(\mathrm{N}, \%)\end{array}$ & $0(0.0)$ & $22(19.0)$ & $0.02 *$ \\
\hline VA Antidepressant Prescription Pre-pregnancy (N, \%) & $6(23.1)$ & $51(44.0)$ & 0.04 \\
\hline VA Antidepressant Prescription during 280-Day Pregnancy Window (N, \%) & $3(11.5)$ & $47(40.5)$ & 0.004 \\
\hline $\begin{array}{l}\text { Any VA Mental Health Care during Pregnancy }(1+\text { mental health visit or } \\
\text { antidepressant prescription in VA CDW EHR) }(\mathrm{N}, \%)\end{array}$ & $11(42.3)$ & $88(75.9)$ & 0.0002 \\
\hline
\end{tabular}

*P value from Fisher's exact test for categorical variables; Satterthwaite test for unequal variances for continuous variables. **Any mental health condition variable includes self-reported ever diagnosis of any of the following: depression, anxiety, PTSD, mood disorder, ADHD/ADD, bipolar disorder, schizophrenia, eating disorder

Time periods: Pre-pregnancy $=($ Newborn date of birth-280)-280; Pregnancy $=$ Newborn date of birth-280 $n=7$ participants were missing $C D W$ data

or antidepressant prescription increased slightly when comparing our 200- and 280-day windows $(63.7 \%$ vs. $73.3 \%)$ but remained the same when comparing 280-day and 300-day windows (both 73.3\%) (Online Resource E).

\section{Qualitative Results}

We identified themes that captured the major concepts surrounding medication use during pregnancy, including themes relevant to mental health medication risk discussions with providers, use of mental health medications during pregnancy, and discontinuation of mental health medications during pregnancy (Table 4). 


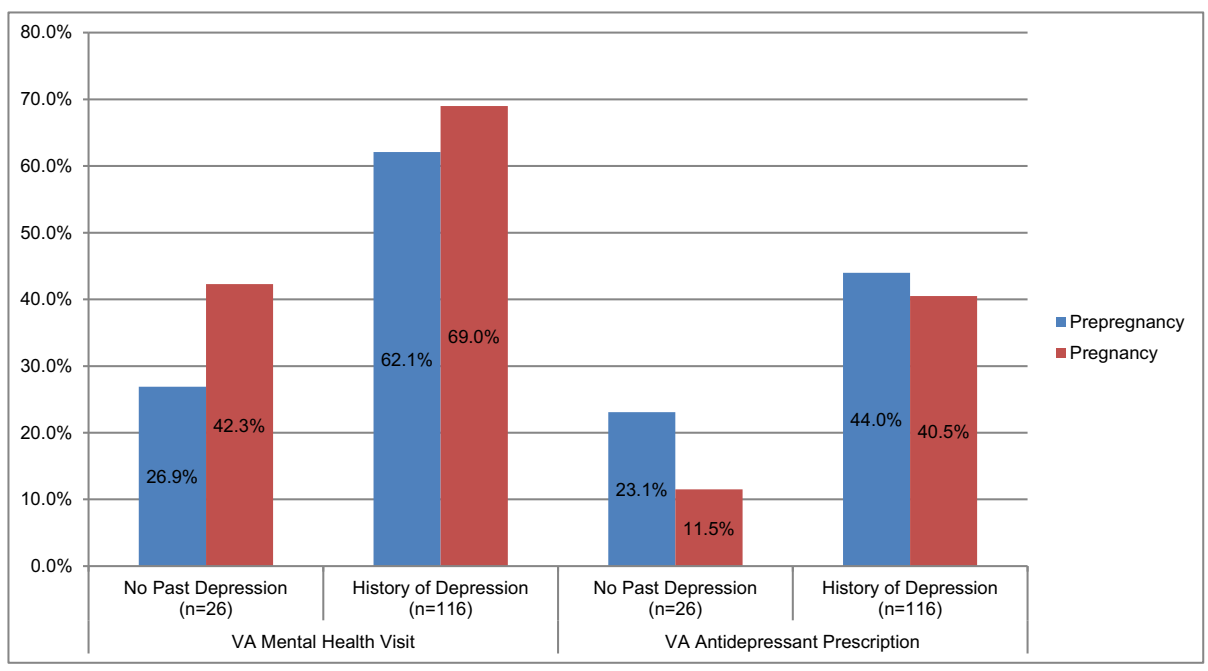

Fig. 2 Mental Health Treatment Utilization*, by Pregnancy Period and Self-Reported Past Depression Diagnosis ( $\mathrm{n}=$ 135). *Mental Health Treatment Utilization: 1+ mental health visit or 30-day antidepressant prescription in VA Corporate Data Warehouse Electronic Health Record Data. Note: 7 of our participants ( $n=1$ with no past depression; $n=6$ with past depression) did not have CDW data; therefore this analysis was conducted in 135 participants

“Once I knew, I stopped" Many women reported discontinuing their medications when they found out they were pregnant. As one woman put it: "Once I knew, I stopped taking everything...I just went off, completely off of everything, everything." One woman who had a planned pregnancy noted that she was trying to "do better as far as managing (her depression)" to "prevent" her from going back on medication while pregnant. Another woman stated that she "kind of quit taking" her antidepressants, but later informed her mental health provider and came up with alternative treatment plans.

Most participants indicated they made the decision to stop on their own. A number of participants reported not feeling comfortable with medication in general ("I'm not a huge believer in long term medicated management for mental health"), and even more so during pregnancy. Very few women in our sample expressed awareness of the risks of abruptly discontinuing their medications, although one participant did share her fears about the potential risks of being on medication and then subsequently weaning herself off too quickly.

A Coordinated Decision Because the COMFORT prenatal interview took place following the participant's visit with her VA provider for pregnancy confirmation, an opportunity for medication review and risk discussion existed. While many participants reported such discussions with their VA providers or their obstetricians, collaboration between VA providers and obstetricians was minimally reported and most participants were unsure whether information about mental health status was being transmitted between their VA and obstetric providers. Those who spoke about coordinated discussions reported conferences with their OB and mental health providers to reach a consensus on a treatment plan during pregnancy. One participant spoke about her mental health provider collaborating with her obstetrician to the extent of changing a medication so that both providers and the patient were comfortable with the treatment: 


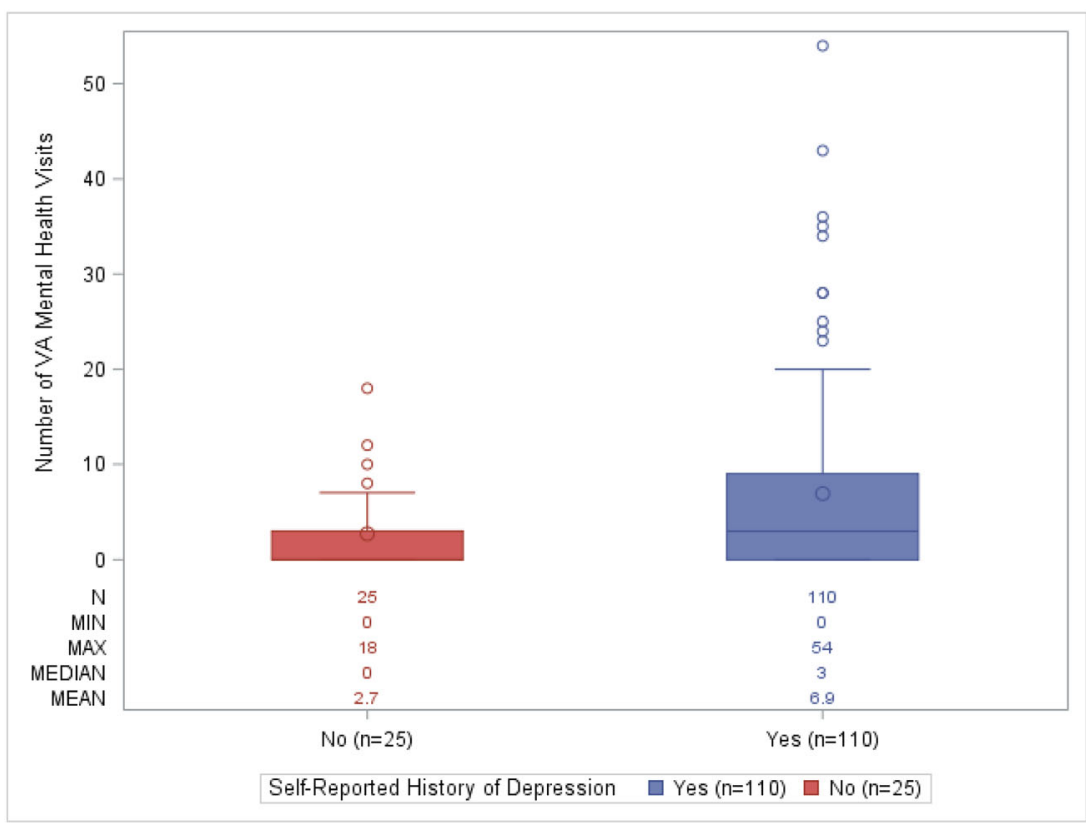

Fig. 3 VA Mental Health Visits* Recorded in the 280 Days Prior to Delivery, by Self-Reported Past Depression Diagnosis, among Pregnant Veterans with Depression Symptoms $(n=135)$. *As recorded in the VA Corporate Data Warehouse Electronic Health Record Data. Note: 7 of our participants ( $n=1$ with no past depression; $n=6$ with past depression) did not have CDW data; therefore this analysis was conducted in 135 participants

"The VA had done a great job of providing peer reviewed information saying their decision about why they think that certain medications are safe during pregnancy. And when that, I think professional opinion, didn't align with what the OBGYN thought they were very happy to basically concede and change the prescription."

Another woman expressed satisfaction with the coordinated decision between her VA provider and obstetrician to have her remain on her medication during pregnancy:

Table 4 Categories, Example Codes, and Themes Identified from Qualitative Interviews with Pregnant Veterans with Depression Symptoms $(n=42)$

\begin{tabular}{|c|c|c|}
\hline Category & Example Codes & Themes \\
\hline $\begin{array}{l}\text { Medication Risk Discussion } \\
\text { with Providers }\end{array}$ & $\begin{array}{l}\text { - Communication between obstetrician } \\
\text { and VA provider } \\
\text { - Occurred with obstetrician } \\
\text { - Occurred with VA provider } \\
\text { - Personal understanding of risks }\end{array}$ & A Coordinated Decision \\
\hline $\begin{array}{l}\text { Use of Medications during } \\
\text { Pregnancy }\end{array}$ & $\begin{array}{l}\text { - Continued medication use } \\
\text { - New medication use }\end{array}$ & Justifying Treatment \\
\hline $\begin{array}{l}\text { Discontinuation of Medications } \\
\text { during Pregnancy }\end{array}$ & $\begin{array}{l}\text { - Alternative treatments } \\
\text { - Feelings about stopping } \\
\text { - Personal decision } \\
\text { - Provider decision }\end{array}$ & $\begin{array}{l}\text { "Once I knew, I stopped" } \\
\text { Not Worth the Risk } \\
\text { Difficulties Adjusting to Life } \\
\text { without Medication }\end{array}$ \\
\hline
\end{tabular}


"She (VA nurse practitioner) was wonderful, she actually looked up the medications because she knew that I had gotten on the medications before I was breastfeeding. So we were very careful about getting on it and I was postpartum from my prior pregnancy, because I had back to back pregnancies they (VA NP and OB) both agreed about keeping me on the medication. She (VA NP) asked me to make sure that my OB was on the same page. And he already was so she was more than helpful. She actually went above and beyond."

However, many participants reported not having a discussion with either their VA mental health providers or their obstetricians, or having a discussion but remaining confused about potential risks during pregnancy. As one woman said:

"I think it was kind of a mutual decision, I had her tell me all of the risks and she went over all, you know, the different studies or whatever that she had pulled up and it just... I just thought (medication) sounded dangerous and it passed through milk and things like that, so I said I definitely wanted to get off (of) that."

Another woman shared that her VA primary care provider was not helpful when she asked about a mental health medication she was taking, and that she had done her own research to decide to discontinue her medication:

"I did my research online as far as all that went, like I said, when I (tried) to talk to my primary care...they really didn't have anything for me as far as ... medical advice...nothing."

Not Worth the Risk Few women reported use of mental health medications during pregnancy. Women who were hesitant to start or restart a medication explained that potential risks to the fetus influenced their decision. Women spoke about medication being "very dangerous" and risky even if their providers suggested a prescription and assured them the risks were minimal. One participant said, "I read all the manufacturing and the risks and everything and some of them said don't take while you're pregnant and so I didn't want to have any risks associated with it." Another participant commented, "I just don't take them. I stopped taking them, just because I didn't want any developmental issues or whatever with the baby." Additionally, one woman added:

"Yeah, she's (provider) even mentioned like taking some antidepressants, but I told her, you know, I'm not a big person on medicine as it is, so I don't like to take medicine, especially like when I'm pregnant...You know because I just don't want to take the risk."

One participant discussed stopping due to past experiences:

"Well, I was taking (medications) for mental health before I got pregnant, then I stopped when I found out I was pregnant cause I don't know, you know, how would that affect the pregnancy, cause I already had two previous miscarriages before this baby."

Justifying Treatment Women who spoke about taking medications during pregnancy seemed to include reasons for justifying their use. One woman spoke about her emotions being to "the extremes":

"(My emotions) were either really sad, tired, angry; where I either wanted to sit in bed all day or sit in the shower and cry all day, or if I was angry, I mean it was to the verge of wanting to hurt others. So my OB/GYN she put me (on) the generic brand of Zoloft... it's helped drastically...I wasn't on any medications whatsoever until I got pregnant." 
Another woman who discontinued her medication during the first trimester but restarted later in pregnancy noted potential changes to her hormones as being the cause for returning symptoms:

"I stopped taking my fluoxetine when I found out I was pregnant. And I was, it was after I was twelve weeks that I started taking it again. I'm not sure the exact week but I remember the doctor said that after twelve weeks it would probably be okay for me to start taking it again. So things got a little bit rough...I don't know if it was the hormones that were a little more crazy but it definitely wasn't very good."

Difficulties Adjusting to Life without Medication Many women reported difficulties adjusting to life without medications to manage their depression. As one woman put it, she had to accept that her depression "is going to be an occasional problem until after I have the kid." Others expressed that techniques to help manage depression were not as effective during pregnancy, or that traveling to mental health appointments had become more difficult since becoming pregnant. One woman said that although she was grateful for the techniques she learned in therapy, without medication "it's just tough, it's really tough to manage that and just, you know, go almost a year without having any medication for it." Very few women discussed starting therapy or utilizing other forms of treatment in place of antidepressants. One woman shared her hesitation about seeking care:

"Well, to be honest, all the things I've been through since I've been pregnant (has been with) my OBGYN...I haven't really spoke with my (VA) primary care provider or I haven't spoke with the therapist there...I don't know, like, and I know this might sound crazy, but it's like I don't feel too comfortable, I guess, discussing it, or I don't know how to bring it up, or stuff like that. I don't know."

\section{Discussion}

To our knowledge, this is the first study to examine mental health care and depression treatment during pregnancy in veterans with depression symptomatology. More than half of our sample had at least one mental health visit at the VA or received a prescription for an antidepressant in the 280-days prior to delivery thus corresponding with the pregnancy period. However, nearly a quarter of our sample did not receive any VA mental health care during their pregnancy. The majority of our participants (82\%) reported using antidepressants at any time prior to their pregnancies, yet only $35 \%$ our sample filled a prescription for an antidepressant in the 280-days prior to delivery. We expected a large proportion of our sample would discontinue use because past research suggests women taking antidepressants prior to pregnancy discontinue use in the prenatal period [37]. Given that treatment for depression will likely decrease further following the birth of the child when time and childcare become barriers to treatment [10], that medication recommendations change over the perinatal period, and that over half of perinatal depression cases develop prior to the postpartum period [38], identifying at-risk and symptomatic women during pregnancy is key to developing a practical treatment routine.

We found that women self-reporting a history of depression were more likely to receive mental health care during their pregnancy, in line with previous work that showed women veterans without a pre-pregnancy mental health diagnosis were less likely to seek outpatient 
mental healthcare during their pregnancies compared to women veterans with a pre-pregnancy mental health diagnosis [39]. Most women who received care in the pre-pregnancy period remained in care during pregnancy; however, women without past diagnoses may potentially be overlooked if depression screening is not done on a routine basis. The women veterans in our analysis who did not report a history of a depression diagnosis comprised $5 \%$ of all pregnant veterans in our sample with a prenatal EPDS $\geq 10$, suggestive of depression symptomatology [3].

The frequency of care utilization among women veterans in our sample differed considerably between women with a self-reported history of depression compared to those with no selfreported history of depression. The VA Maternity Health Care and Coordination Handbook defines a standard of practice for maternity care coordination within VA medical facilities, instituted to bridge the gap between existing dual VA/non-VA healthcare systems and designed to "ensure that eligible women Veterans have access to timely prenatal care...(and) seamless coordination of non-VA maternity care with VA care, especially in cases of women Veterans with co-morbid conditions who may require VA care during their pregnancy" [5]. These guidelines also recommend depression assessment, screening, counseling, and referral for care as components of the coordination model. The guidelines specify that "VA providers must screen all pregnant and postpartum women for depression utilizing an appropriate screening tool, and have a system to ensure that positive screening results are followed by accurate diagnosis, implementation of treatment, and follow-up either within the practice or through referral" [5]. Given our quantitative findings showing that women without a history of depression were less likely to utilize care during pregnancy, conducting early and frequent depression screening, as well as establishing referral processes and introducing women without a history of mental health care to the mental health resources at the VA is key for this population. Our results indicate that veterans experiencing depression symptoms may be lacking access to treatment, despite guidelines encouraging systems to identify these women and establish a care routine.

Veterans in our sample qualitatively reported varying experiences with mental health care treatment during pregnancy. While many women reported discontinuing medications after discussions of associated risks with providers, several women reported discontinuing medications on their own, without a risk/benefit discussion with their providers. This is consistent with other work showing that, in general, women tend to make a decision to discontinue medications during pregnancy without the benefit of a full discussion with their providers [40]. A lack of discussion between patients and providers may also be suggestive of reluctance that provider's may have with discussing and prescribing psychotropic medication during pregnancy $[7,41]$, despite patients preferring their providers initiate the discussion about medication risks during pregnancy [42]. Additionally, as has been reported in similar studies, the fear of harming the fetus often results in medication discontinuation during pregnancy [43, 44]. The stigma surrounding medication use during pregnancy is pervasive. Previous work has found pregnant women expressing difficulties in accepting the need for a medication during pregnancy [40] given the prevalent focus on the health of the fetus as priority over the health of the mother $[45,46]$ and without recognition of the risks of untreated mental health conditions being associated with significant risk for mother and child. Our sensitivity analyses showed a greater number of antidepressant prescriptions in the 280-day and 300-day windows compared to the 200-day window when looking back from the date of delivery or estimated due date; this may be quantitative evidence that many women are discontinuing their antidepressant prescriptions early in pregnancy. 
Our study is not without limitations. It is possible that women started taking an antidepressant or seeing a mental health provider following the prenatal interview, which ranged from 4 to 39 weeks gestation in our sample, and thus would not be captured in the self-report responses. Outside of the prenatal interview question specifically asking about antidepressant use prior to pregnancy, our interview included broad questions regarding self-reported receipt of mental health care rather than solely focusing on depression. Additionally, we may have included antidepressant prescriptions or mental health visits from the CDW data outside the actual pregnancy window, such as prescriptions filled in very early pregnancy which may have been stopped upon pregnancy confirmation. However, our sensitivity analyses using varying pregnancy windows of 200 and 300 days showed expected differences of increases in mental health visits and decreases in antidepressant prescriptions when our pregnancy time was varied. Likewise, we were unable to identify mental health care received during pregnancy from a non-VA provider, which may explain our low specificity calculations. Given that most discordant cases were due to women selfreporting depression where it was shown absent in the EHR, this could potentially be due to diagnoses outside of VA care (either before the veteran was receiving VA care or made by a provider outside of the VA system). We also only included antidepressant prescriptions in our analysis. We chose to focus on depression symptomology for this paper; however, this potentially excludes a wide range of other mental health medications our participants may have been taking. Finally, our findings are not generalizable to a population outside of English-speaking pregnant veterans receiving their care within the VA medical system.

Our study also has several strengths. We enriched our survey dataset with EHR data and examined the sensitivity and specificity of our participants self-reported history of depression diagnoses compared to EHR records. We used both quantitative and qualitative data to enrich our understanding of mental health care of pregnant veterans. We utilized a large, national sample to enroll participants. Additionally, we used the EPDS which has been validated as a screening tool for use in a pregnant population (Bergink et al., 2011). Finally, our qualitative data was obtained from semi-structured interviews, which gave the participants flexibility in their responses.

\section{Conclusions}

Estimates of depression prior to pregnancy in women veterans range from 10 to $60 \%$, suggesting a higher likelihood of pregnant veterans developing depression compared to pregnant women in the general population [1,47], and depression is the most commonly diagnosed medical condition among all women veterans ages 18-44 [48]. The US Preventive Services Task Force (USPSTF) recently issued a draft recommendation that clinicians "provide or refer pregnant and postpartum women who are at increased risk of perinatal depression to counseling interventions" [49]. These recommendations are based on lack of current guidelines for preventing perinatal depression, in light of evidence showing that counseling interventions can help improve perinatal depression outcomes. The USPSTF recommends identifying women at-risk for perinatal depression early, based on risk factors such as a history of depression, current depressive symptoms, and socioeconomic risk factors. Women veterans, especially given their increased risk of depression, could benefit from preventative therapy and other interventions targeting awareness around the importance of treating depression during pregnancy. These discussions should include nonpharmacologic and pharmacologic treatment options including balanced risk discussion addressing outcomes associated with untreated and 'treated disease. Our study reveals that treatment and care experiences vary among women veterans substantially. Women veterans without a history of 
depression may be less likely to receive care for depression symptoms during pregnancy. However, a majority of our sample with prenatal depression symptoms had at least one mental health visit or an anti-depression medication fill during their pregnancy window, suggesting that mental health care is available for women veterans when accessed.

\section{Implications for Practice and/or Policy}

Further work needs to be done to understand the extent of depression screening, medication adherence, and treatment options among veterans in the perinatal period, especially for pregnant women with new onset symptoms who may not have had a previous encounter with VA mental health. Consistency in care, such as therapy for prevention of depressive symptomatology and standards of practice for medication risk discussions, could help veterans and providers better manage depression during pregnancy as well as encourage long-term treatment.

Acknowledgements This material is based on work supported by the Department of Veterans Affairs, Veterans Health Administration, Office of Research and Development (HSR\&D IIR13-81). All authors had full access to all of the data in the study and take responsibility for the integrity of the data and the accuracy of the data analysis. The views expressed in this article are those of the authors and do not reflect the position or policy of the Department of Veterans Affairs or the United States Government. The authors thank Laurel A. Copeland, PhD, for her assistance with the analysis.

\section{Compliance with Ethical Standards}

All procedures performed in studies involving human participants were in accordance with the ethical standards of the institutional and/or national research committee and with the 1964 Helsinki declaration and its later amendments or comparable ethical standards. This article does not contain any studies with animals performed by any of the authors.

Conflict of Interest Dr. Moore Simas receives grant funding from the National Institute of Health (R41 MH113381) and the Centers for Disease Control and Prevention (U01DP006093) for projects related to perinatal depression. Dr. Moore Simas receives a stipend from the Massachusetts Department of Mental Health via Beacon for her role as Engagement Director of the Massachusetts Child Psychiatry Access Program for Moms (MCPAP for Moms). Dr. Moore Simas is Medical Director of Lifeline4Moms; she co-directs the American College of Obstetricians and Gynecologists' Expert Work Group on Maternal Mental Health and was a member of the Council on Patient Safety in Women's Health Care's task force for creation of the maternal mental health patient safety bundle and co-author on the associated commentary. Dr. Moore Simas has served on ad hoc Physician Advisory Boards for Sage Therapeutics, has received speaking honoraria, and serves as a consultant on observational studies and a systematic review. Dr. Moore Simas serves as a consultant to Ovia Health, has received compensation for reviewing a perinatal depression case for McGraw Hill, and has received speaking honoraria from Miller Medical Communications. All other authors declare that they have no conflict of interest.

\section{References}

1. Mattocks KM, Skanderson M, Goulet JL, et al. Pregnancy and mental health among women veterans returning from Iraq and Afghanistan. J Women's Health. 2010;19(12):2159-66. https://doi.org/10.1089/jwh.2009.1892.

2. Shivakumar G, Anderson EH, Surís AM. Managing posttraumatic stress disorder and major depression in women veterans during the perinatal period. J Women's Health. 2015;24:18-22.

3. Kroll-Desrosiers, AR, Crawford SL, Moore Simas, TA, Clark, MA, Bastian, LA, \& Mattocks, K. M. Rates and Correlates of Depression Symptoms in a Sample of Pregnant Veterans Receiving Veterans Health Administration Care. Womens Health Issues. 2019;29(4):333-40. https://doi.org/10.1016/j. whi.2019.04.008. 
4. Moore Simas TA, Flynn MP, Kroll-Desrosiers AR, et al. A systematic review of integrated care interventions addressing perinatal depression care in ambulatory obstetric care settings. Clin Obstet Gynecol. 2018;61(3):57390. https://doi.org/10.1097/GRF.0000000000000360.

5. Department of Veterans Affairs (2012) VHA Handbook 1330.03, Maternity Health Care and Coordination.

6. West AN, Lee PW. Associations between childbirth and women veterans' VA and non-VA hospitalizations for major diagnostic categories. Mil Med. 2013;178:1250-5.

7. Byatt N, Biebel K, Lundquist RS, et al. Patient, provider, and system-level barriers and facilitators to addressing perinatal depression. J Reprod Infant Psychol. 2012;30(5):436-449. https://doi.org/10.1080 /02646838.2012.743000.

8. Hofmann SG, Asnaani A, Vonk IJJ, Sawyer AT, Fang A. The efficacy of cognitive behavioral therapy: a review of Meta- analyses. Cognit Ther Res. 2012;36:427-40.

9. Vigod SN, Wilson CA, Howard LM. Depression in pregnancy. BMJ. 2016;352:i1547.

10. Byatt N, Moore Simas TA, Lundquist RS, Johnson J V., Ziedonis DM. Strategies for improving perinatal depression treatment in North American outpatient obstetric settings. J Psychosom Obstet Gynecol. 2012;33(4):143-61. https://doi.org/10.3109/0167482X.2012.728649.

11. Alwan S, Reefhuis J, Rasmussen SA, Friedman JM. Patterns of antidepressant medication use among pregnant women in a United States population. J Clin Pharmacol. 2011;51:264-70.

12. Yamamoto A, McCormick MC, Burris HH. Disparities in antidepressant use in pregnancy. J Perinatol. 2015;35:246-51.

13. Cooper WO, Willy ME, Pont SJ, Ray WA (2007) Increasing use of antidepressants in pregnancy. Am J Obstet Gynecol 196:544.e1-544.e5.

14. Schwarz EB, Brandt C, Borrero S, Zephyrin LC, Bathulapalli H, Haskell S. Counseling of female veterans about risks of medication-induced birth defects. J Gen Intern Med. 2013;28(Suppl 2):S598-603.

15. Yonkers KA, Wisner KL, Stewart DE, Oberlander TF, Dell DL, Stotland N, et al. The management of depression during pregnancy: a report from the American Psychiatric Association and the American College of Obstetricians and Gynecologists. Obstet Gynecol. 2009;114:703-13.

16. Field T. Postpartum depression effects on early interactions, parenting, and safety practices: a review. Infant Behav Dev. 2010;33:1-6.

17. Miller LJ. Postpartum depression. JAMA. 2002;287:762-5.

18. Department of Veterans Affairs; Department of Defense (2016) VA/DoD clinical practice guideline for the management of major depressive disorder clinician guideline summary qualifying statements.

19. American College of Obstetricians and Gynecologists (2012) Depression (FAQ106).

20. Byatt N, Levin LL, Ziedonis D, Moore Simas TA, Allison J. Enhancing participation in depression Care in Outpatient Perinatal Care Settings: a systematic review. Obstet Gynecol. 2015;126(5):1048-58. https://doi. org/10.1097/AOG.0000000000001067.

21. Mattocks KM, Baldor R, Bean-Mayberry B, Cucciare M, Gerber MR, Goldstein, KM, et al. Factors Impacting Perceived Access to Early Prenatal Care among Pregnant Veterans Enrolled in the Department of Veterans Affairs. Womens Health Issues, 2019;29(1):56-63. https://doi.org/10.1016/j.whi.2018.10.001.

22. Harris PA, Taylor R, Thielke R, Payne J, Gonzalez N, Conde JG. Research electronic data capture (REDCap)-a metadata-driven methodology and workflow process for providing translational research informatics support. J Biomed Inform. 2009;42:377-81.

23. Department of Veterans Affairs (2014) 172VA10P2: VHA Corporate Data Warehouse-VA. 79 FR 4377. Austin

24. Skanderson M, Bastian LA, Brandt CA, Haskell S, Kerns RD. Receipt of prescription opioids in a National Sample of pregnant veterans receiving veterans health administration care. Womens Health Issues. 2015;26(2):240-6. https://doi.org/10.1016/j.whi.2015.09.010.

25. Babb JA, Nephew BC, Guilarte-Walker Y, Deligiannidis KM. Association of peripartum synthetic oxytocin administration and depressive and anxiety disorders within the first postpartum year. Depress Anxiety. 2017;34:137-46. https://doi.org/10.1002/da.22599.

26. Department of Veterans Affairs (2013) VHA Directive 1731: Decision Support System Outpatient Identifiers.

27. Flynn HA, Blow FC, Marcus SM. Rates and predictors of depression treatment among pregnant women in hospital-affiliated obstetrics practices. Gen Hosp Psychiatry. 2006;28:289-95.

28. Cox JL, Holden JM, Sagovsky R. Detection of postnatal depression. Development of the 10-item Edinburgh postnatal depression scale. Br J Psychiatry. 1987;150:782-6.

29. Bergink V, Kooistra L, Lambregtse-van den Berg MP, Wijnen H, Bunevicius R, van Baar A, et al. Validation of the Edinburgh depression scale during pregnancy. J Psychosom Res. 2011;70:385-9.

30. Matthey S, Henshaw C, Elliott S, Barnett B. Variability in use of cut-off scores and formats on the Edinburgh postnatal depression scale - Implications for clinical and research practice. Arch Womens Ment Health. 2006;9:309-15. 
31. Kimerling R, Gima K, Smith MW, Street A, Frayne S. The veterans health administration and military sexual trauma. Am J Public Health. 2007;97:2160-6.

32. Frayne SM, Phibbs CS, Friedman SA, et al (2012) Sourcebook: women veterans in the veterans health administration. Volume 2. Sociodemographics and use of VHA and non-VA care (fee). Washington DC.

33. Smith JA, Osborn M (2008) Interpretative phenomenological analysis. In: Smith JA (ed) Qual. Psychol. A Pract. Guid. To res. Methods, second. SAGE publications, London, pp 53-80.

34. Creswell JW. Qualitative inquiry and research design: choosing among five approaches. Thousand Oaks: SAGE Publications; 2007.

35. Colaizzi PF. Psychological research as the phenomenologist views it. In: Valle RS, Mark K, editors. Existent. New York: Phenomenol. Altern. Psychol. Oxford University Press; 1978. p. 48-71.

36. Mattocks KM, Haskell SG, Krebs EE, Justice AC, Yano EM, Brandt C. Women at war: understanding how women veterans cope with combat and military sexual trauma. Soc Sci Med. 2012;74(4):537-45. https://doi.org/10.1016/j.socscimed.2011.10.039.

37. Bennett IM, Marcus SC, Palmer SC, Coyne JC. Pregnancy-related discontinuation of antidepressants and depression care visits among Medicaid recipients. Psychiatr Serv. 2010;61:386-91.

38. Wisner KL, Sit DKY, McShea MC, Rizzo DM, Zoretich RA, Hughes CL, et al. Onset timing, thoughts of self-harm, and diagnoses in postpartum women with screen-positive depression findings. JAMA Psychiat. 2013;70:490-8.

39. Katon JG, Lewis L, Hercinovic S, McNab A, Fortney J, Rose SM. Improving perinatal mental health Care for Women Veterans: description of a quality improvement program. Matern Child Health J. 2017;21:1598605.

40. Lynch MM, Squiers LB, Kosa KM, et al. Making decisions about medication use during pregnancy: Implications for communication strategies. Matern Child Health J. 2017;22:92-100.

41. Weinreb L, Byatt N, Moore Simas T, Tenner K, Savageau J. What happens to mental health treatment during pregnancy? Women's experience with prescribing providers. Psychiatr Q. 2014;85(3):349-55. https://doi.org/10.1007/s11126-014-9293-7.

42. Santucci AK, Gold MA, Akers AY, Borrero S, Schwarz EB. Women's perspectives on counseling about risks for medication-induced birth defects. Birth Defects Res Part A - Clin Mol Teratol. 2010;88:64-9.

43. Lynch MM, Amoozegar JB, McClure EM, Squiers LB, Broussard CS, Lind JN, et al. Improving safe use of medications during pregnancy: the roles of patients, physicians, and pharmacists. Qual Health Res. 2017;27: 2071-80.

44. Bennett HA, Boon HS, Romans SE, Grootendorst P. Becoming the best mom that I can: Women's experiences of managing depression during pregnancy - a qualitative study. BMC Womens Health. 2007; 7:1-14.

45. Lupton D. 'Precious cargo': foetal subjects, risk and reproductive citizenship. Crit Public Health. 2012;22: 329-40.

46. Hammer RP, Burton-Jeangros C. Tensions around risks in pregnancy: a typology of women's experiences of surveillance medicine. Soc Sci Med. 2013;93:55-63.

47. Haskell SG, Goulet JL, Krebs EE, Skanderson M, Leslie D, Justice AC, et al. The burden of illness in the first year home: do male and female VA users differ in health conditions and healthcare utilization. Womens Health Issues. 2011;21:92-7.

48. Frayne SM, Phibbs CS, Saechao F, et al (2014) Sourcebook: women veterans in the veterans health administration. Volume 3: Sociodemographics, utilization, costs of care, and health profile. Washington DC.

49. US Preventive Services Task Force (2018) Draft Recommendation Statement: Perinatal Depression: Preventive Interventions. https://www.uspreventiveservicestaskforce.org/Page/Document/draftrecommendation-statement/perinatal-depression-preventive-interventions. Accessed 4 Sep 2018.

Publisher's Note Springer Nature remains neutral with regard to jurisdictional claims in published maps and institutional affiliations.

Aimee R. Kroll-Desrosiers, $\mathrm{PhD}$, is a Research Health Scientist at the VA Central Western Massachusetts and an Assistant Professor in the Department of Population and Quantitative Health Sciences at the University of Massachusetts Medical School.

Sybil L. Crawford , PhD, is a Professor in the Graduate School of Nursing and adjunct faculty in the Clinical and Population Health Research program at the University of Massachusetts Medical School. 
Tiffany A. Moore Simas, MD, MPH, MEd, FACOG, is Associate Professor of Obstetrics \& Gynecology and Pediatrics at the University of Massachusetts Medical School and Director of the Obstetrics \& Gynecology Research Division.

Melissa A. Clark, PhD, is Professor of Population and Quantitative Health Sciences in the Division of Epidemiology of Chronic Diseases at the University of Massachusetts Medical School.

Kristin M. Mattocks, PhD, MPH, is Associate Chief of Staff for Research and Education at VA Central Western Massachusetts Healthcare System and Associate Professor of Population and Quantitative Health Sciences and Psychiatry at the University of Massachusetts Medical School. 